\title{
The Flavonoid Jaceosidin from Artemisia princeps Induces Apoptotic Cell Death and Inhibits the Akt Pathway in Oral Cancer Cells
}

\author{
Hye-Yeon Han, ${ }^{1}$ Hyung Joon Kim, ${ }^{2}$ Seung-Hwa Jeong, ${ }^{3}$ Jiyeon Kim, ${ }^{4}$ Sung-Hee Jeong, ${ }^{5}$ \\ Gyoo Cheon Kim, ${ }^{6}$ Dae-Seok Hwang, ${ }^{7}$ Uk-Kyu Kim, ${ }^{7}$ and Mi Heon Ryu ${ }^{8}{ }^{8}$
}

${ }^{1}$ Department of Oral Pathology, School of Dentistry, Research Institute for Oral Biotechnology, Pusan National University, Yangsan, Gyeongnam 50612, Republic of Korea

${ }^{2}$ Department of Oral Physiology, BK21 Plus Project and Institute of Translational Dental Sciences, School of Dentistry, Pusan National University, Yangsan, Gyeongnam 50612, Republic of Korea

${ }^{3}$ Department of Preventive and Community Dentistry, BK21 Plus Project, School of Dentistry, Pusan National University, Yangsan, Gyeongnam 50612, Republic of Korea

${ }^{4}$ Department of Pediatric Dentistry, School of Dentistry, Dental Research Institute, Pusan National University, Yangsan, Gyeongnam 50612, Republic of Korea

${ }^{5}$ Department of Oral Medicine, School of Dentistry, Pusan National University, Dental Research Institute, Yangsan, Gyeongnam 50612, Republic of Korea

${ }^{6}$ Department of Dental Anatomy, BK21 Plus Project, School of Dentistry, Pusan National University, Yangsan, Gyeongnam 50612, Republic of Korea

${ }^{7}$ Department of Oral and Maxillofacial Surgery, School of Dentistry, Pusan National University, Yangsan, Gyeongnam 50612, Republic of Korea

${ }^{8}$ Department of Oral Pathology, BK21 Plus Project, School of Dentistry, Pusan National University, Yangsan, Gyeongnam 50612, Republic of Korea

Correspondence should be addressed to Mi Heon Ryu; apollon@pusan.ac.kr

Received 13 February 2018; Accepted 5 April 2018; Published 13 May 2018

Academic Editor: Solomon Habtemariam

Copyright (C) 2018 Hye-Yeon Han et al. This is an open access article distributed under the Creative Commons Attribution License, which permits unrestricted use, distribution, and reproduction in any medium, provided the original work is properly cited.

Jaceosidin is a single compound from the Japanese mugwort Artemisia princeps, which is used as a food and a traditional medicinal herb. A. princeps extracts and flavonoid components have been shown to have antihyperglycaemic, antioxidant, and antiinflammatory properties. Although the anticancer properties of these extracts were recently demonstrated, the related mechanisms have not been characterised. In this study, we investigated the effects of jaceosidin in oral squamous cell carcinoma (OSCC) cells and initially showed selective suppression of proliferation ( $\mathrm{IC}_{50}=82.1 \mu \mathrm{M}$ in HSC-3 cells and $97.5 \mu \mathrm{M}$ in Ca9.22 cells) and accumulation of cells at the sub-G1 stage of the cell cycle. In addition, jaceosidin increased cleavage of caspase- 9 and caspase- 3 in OSCC cells, although caspase- 8 was not detected. In further experiments, jaceosidin downregulated Akt phosphorylation and ectopic activation of Akt blocked the antiproliferative effects of jaceosidin. Finally, we showed that jaceosidin has no effects on HaCaT normal epithelial cell viability, indicating selective chemotherapeutic potential of jaceosidin and that tumour-specific downregulation of Akt increases apoptosis and inhibits growth in OSCC cells.

\section{Introduction}

Oral squamous cell carcinoma (OSCC) accounts for $90 \%$ of oral cavity malignancies, and about 3,000 new OSCC diagnoses were recorded in 2011 in South Korea [1]. Surgical resection and chemotherapy are the prevailing therapeutic strategies for OSCC, and although these treatments have improved over several decades, survival rates of OSCC patients remain relatively low at around 50\% [2]. In addition, defects of head and neck regions following removal of OSCCs 
are aesthetically and functionally debilitating and include mastication problems and difficulties speaking. Chemotherapies for OSCC patients also have severe side effects, such as nausea/vomiting, alopecia, mucositis, headaches, chronic weakness, and exhaustion, and these contribute to low treatment success rates [3]. Therefore, targeted chemotherapeutic agents for OSCC are required to reduce complications and improve 5-year survival rates. Such anticancer agents should primarily act by inducing apoptosis, because dysfunctional apoptotic pathways are the hallmark of all malignancies [4].

Artemisia genus consists of a large and diverse family of plants, and more than 500 Artemisia herbs are widespread worldwide and many are endemic to Korea [4]. Most Artemisia species have been used as food additives and teas and provide important ingredients for various traditional Korean medicines for gynecologic diseases, gastrointestinal conditions, hepatitis, eczema, furuncles, inflammation, and tumours $[4,5]$. Extracts from Artemisia species contain many flavonoids, including jaceosidin, eupalitin, and eupafolin [6], and jaceosidin is considered one of the most active ingredients [7-9].

Few studies demonstrate the antioxidant, anti-inflammatory, and immunosuppressive effects of jaceosidin and inhibition of several human cancers. However, jaceosidin promoted angiogenesis in endothelial cells [10] and had strong antimutagenic activity and anticancer chemotherapeutic potential [9] in previous studies. Furthermore, jaceosidin reportedly inhibited the functions of E6 and E7 oncoproteins of human papillomavirus 16, potentially limiting the proliferation of several human cancer cells $[9,11]$. Accordingly, Chung et al. showed that ethanol extracts of Artemisia inactivate colitisassociated colon tumorigenesis in mice [12]. However, to our knowledge, the effects of jaceosidin on OSCCs have not been investigated to date. Thus, we demonstrated the pharmacological potential and modes of action of jaceosidin in OSCC cells.

\section{Materials and Methods}

2.1. Reagents and Antibodies. Jaceosidin (CAS 18085-97-7) was kindly provided by Professor Hyungwoo Kim of the Pusan National University. Paclitaxel, propidium iodide (PI), DAPI, and 3,4,5-dimethyl-N-methylthiazol-2-yl-2,5-dphenyl tetrazolium bromide (MTT) were purchased from Sigma-Aldrich (St. Louis, MO, USA). Annexin V-FITC apoptosis detection kits were obtained from BD Biosciences (CA, USA) and antibodies against cleaved caspase-3, cleaved caspase-9, cleaved poly(ADP-ribose) polymerase (PARP), Akt, and phospho-Akt were supplied by Cell Signaling Technology (Beverly, MA, USA). Anti-mouse IgG secondary antibody and anti-rabbit IgG secondary antibody were obtained from Enzo Life Sciences (Farmingdale, NY, USA), whereas anti-beta-actin antibody was purchased from Santa Cruz Biotechnology (Santa Cruz, CA, USA).

2.2. Cell Culture. The human OSCC cell lines HSC-3 and Ca9.22 were obtained from the Japanese Collection of Research Bioresources Cell Bank (JCRB Cell Bank). HSC3 and HaCaT cells were cultured in Dulbecco's Modified Eagle's Medium (DMEM) containing 10\% fetal bovine serum and $1 \%$ penicillin/streptomycin in a humidified environment containing $5 \% \mathrm{CO}_{2}$ at $37^{\circ} \mathrm{C}$. Ca9.22 cells were cultured and maintained in Modified Eagle's Medium (MEM, Hyclone, UT, USA) containing 10\% fetal bovine serum (FBS, Hyclone) with $1 \%$ penicillin/streptomycin (Invitrogen, NY, USA) in a humidified chamber containing $5 \% \mathrm{CO}_{2} / 95 \%$ air at $37^{\circ} \mathrm{C}$. Equal numbers of OSCC cells $\left(4 \times 10^{4}\right.$ cells/well) were seeded onto 24-well plates and were used in MTT assays and other analyses as adherent cultures.

2.3. Proliferation Assays. The antiproliferative activities of jaceosidin were determined using MTT assays. Briefly, cells were seeded at $4 \times 10^{4}$ cells per well in 24 -well plates and were allowed to adhere overnight. Jaceosidin was diluted in DMSO and was added to cell cultures at $0,12.5,25,50$, or $100 \mu \mathrm{M}$. After $24 \mathrm{~h}$ jaceosidin treatments, MTT solution $(500 \mu \mathrm{l})$ was added to cells and incubated at $37^{\circ} \mathrm{C}$ for $4 \mathrm{~h}$ in air containing $5 \% \mathrm{CO}_{2}$. Media were then carefully removed after solubilisation of formazan crystals, and optical densities (OD) of the converted dye were measured at $570 \mathrm{~nm}$ using a microplate reader (Bio-Rad Laboratories, Hercules, CA, USA).

2.4. Cell Morphology. To assess morphological changes of HSC-3 and Ca9.22 cells after jaceosidin treatments, cells were analysed and photographed using a phase contrast microscope at 200x magnification (Olympus, Tokyo, Japan).

2.5. Cell Cycle Analyses. Changes in cell cycle progression of jaceosidin-treated OSCC cells were assessed using flow cytometry. In these analyses, HSC-3 and Ca9.22 cells $\left(1 \times 10^{6}\right.$ cells per well) were treated with DMSO (vehicle) or jaceosidin at 25,50 , or $100 \mu \mathrm{M}$ for $48 \mathrm{~h}$. Cells were then harvested and stained with PI solution $(10 \mu \mathrm{g} / \mathrm{ml})$. Cell cycle stages were then evaluated using a FACS Scan flow cytometer (BD Biosciences, Heidelberg, Germany).

2.6. Assessments of Apoptosis. To determine rates of jaceosidin-induced apoptosis, cells were labelled with Annexin VFITC and PI and were then analysed using flow cytometry. Cells were seeded in 6 -well plates at $3 \times 10^{5}$ cells per well and were incubated overnight and then treated with jaceosidin at 25,50 , or $100 \mu \mathrm{M}$ for $48 \mathrm{~h}$. Control cells were treated with vehicle (DMSO) for $48 \mathrm{~h}$ and cells treated with $30 \mathrm{nM}$ paclitaxel for $48 \mathrm{~h}$ were used as positive controls. After treatments, cells were harvested with trypsin, washed with phosphatebuffered saline (PBS) twice, resuspended in $500 \mu \mathrm{L}$ of Binding Buffer (Annexin V-FITC apoptosis detection kit, Enzo), and finally stained using Annexin V-FITC apoptosis detection kits (Enzo) at room temperature for $5 \mathrm{~min}$ in the dark according to the manufacturer's instructions. Stained cells were then analysed using a FACS Scan flow cytometer (BD Biosciences, Heidelberg, Germany), and the data were processed using FACSCanto II software.

2.7. Western Blotting. Following jaceosidin treatments, cell lysates were extracted using RIPA buffer (Cell Signaling Technology) according to the manufacturer's instructions and protein concentrations were determined. Subsequently, $40 \mu \mathrm{g}$ samples were electrophoresed on SDS-polyacrylamide gels in triplicate and were then transferred to polyvinylidene fluoride membranes. After blocking with skim milk, membranes 

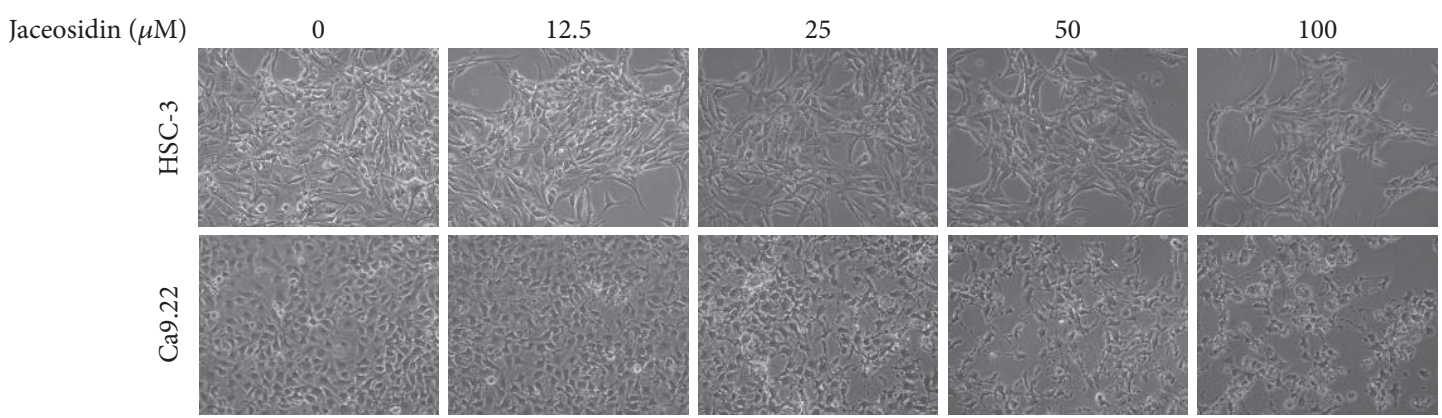

(a)
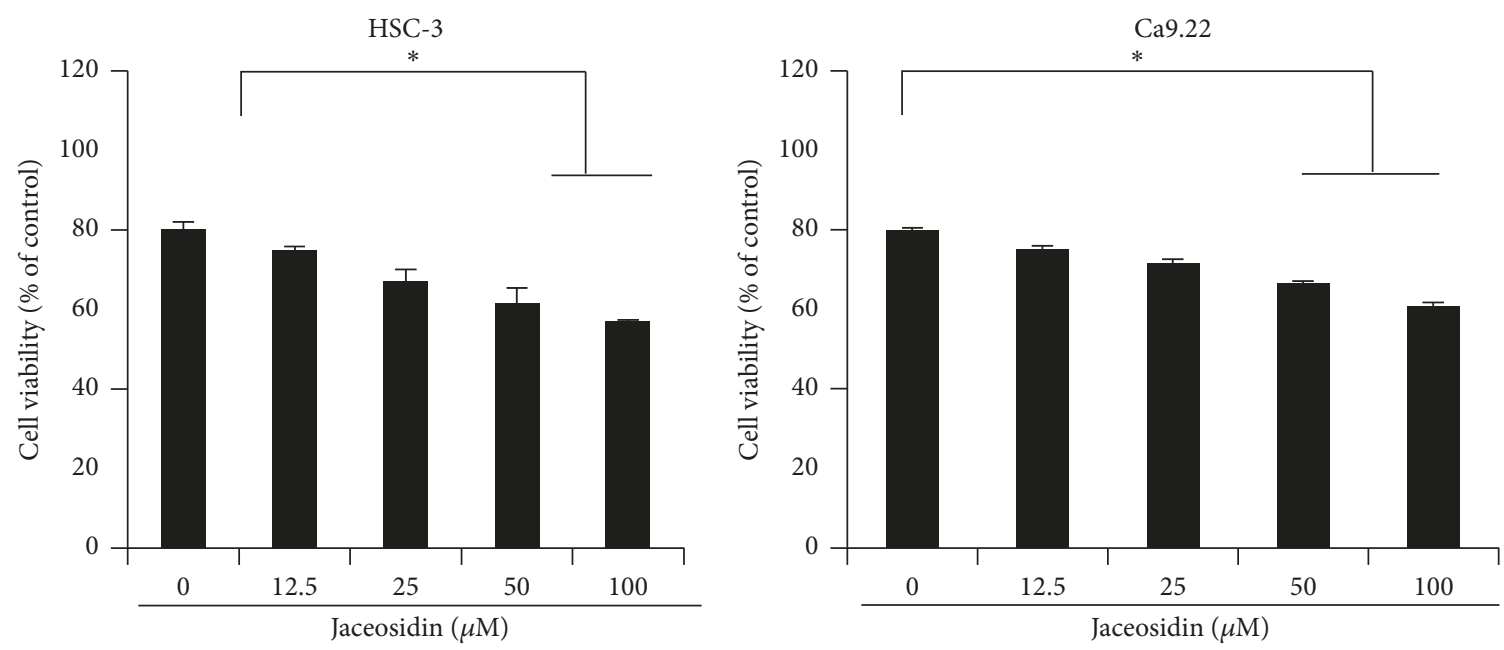

(b)

FIGURE 1: Antiproliferative effects of jaceosidin in OSCC cells; HSC-3 and Ca9.22 OSCC cells were treated with jaceosidin at 0, 12.5, 25, 50, or $100 \mu \mathrm{M}$ for $24 \mathrm{~h}$. (a) Morphologies of jaceosidin-treated HSC-3 cells (top) and Ca9.22 cells (bottom). (b) Percentages of viable cells were determined after $24 \mathrm{~h}$ using MTT assays. The viability of HSC-3 cells (left) and Ca9.22 cells (right); independent experiments were performed in triplicate; ${ }^{*} p<0.05$.

were incubated with primary antibodies against cleaved caspase-3, cleaved caspase-9, cleaved PARP, Akt, phosphoAkt, and beta-actin (internal control) at $4^{\circ} \mathrm{C}$ overnight. Finally, HRP-conjugated secondary antibodies (1:5000) were applied at room temperature and protein signals were detected using SuperSignal West-Femto reagent (Pierce, Rockford, IL, USA).

2.8. Statistical Analysis. Data are presented as means \pm standard deviations (SD). Differences between control and treatment groups were identified using Student's $t$-test (SPSS ver. 21.0, SPSS, Illinois, USA) and were considered significant when $p<0.05$, as indicated with asterisks.

\section{Results}

3.1. Jaceosidin Inhibits OSCC Cell Proliferation. The anticancer effects of jaceosidin against the OSCC cells lines HSC3 and Ca9.22 were evaluated using MTT assays. Jaceosidin inhibited OSCC cell proliferation in a dose-dependent manner (Figures 1(a) and 1(b)), with half inhibitory concentrations $\left(\mathrm{IC}_{50}\right.$ ) of $82.1 \mu \mathrm{g} / \mathrm{mL}$ in HSC-3 cells and $97.5 \mu \mathrm{g} / \mathrm{mL}$ in Ca9.22 cells. In addition, marked morphological changes were evident following treatments of OSCC cells with jaceosidin. Specifically, jaceosidin-treated OSCC cells were smaller and rounder and lost visible cellular processes in a dosedependent manner (Figure 1(a)), suggesting that jaceosidin has antiproliferative activity in oral cancer cells.

3.2. Jaceosidin Induced Early and Late Apoptosis in OSCC Cells and Caused Accumulation of Cells in the Sub-G1 Phase. We performed PI and Annexin V-FITC double staining to investigate the antiproliferative mechanisms of jaceosidin. Jaceosidin treatments for $48 \mathrm{~h}$ significantly increased the number of apoptotic cells compared with those of untreated cells, and these effects were dose-dependent. In particular, $25 \mu \mathrm{M}$ jaceosidin led to prominent early and late apoptosis in HSC-3 and Ca9.22 cells (Figures 2(a) and 2(b)), and marked increases in $\mathrm{PI} /$ Annexin $\mathrm{V}$ double positive cell numbers were observed relative to PI-negative/Annexin V-positive cell numbers. In addition, $100 \mu \mathrm{M}$ jaceosidin treatments led to marked increases in percentages of cells in the sub-G1 cell cycle stage (Figures 3(a) and 3(b)), with $23.8 \%$ and $45.6 \%$ subG1 accumulations of HSC-3 and Ca9.22 cells, respectively. 

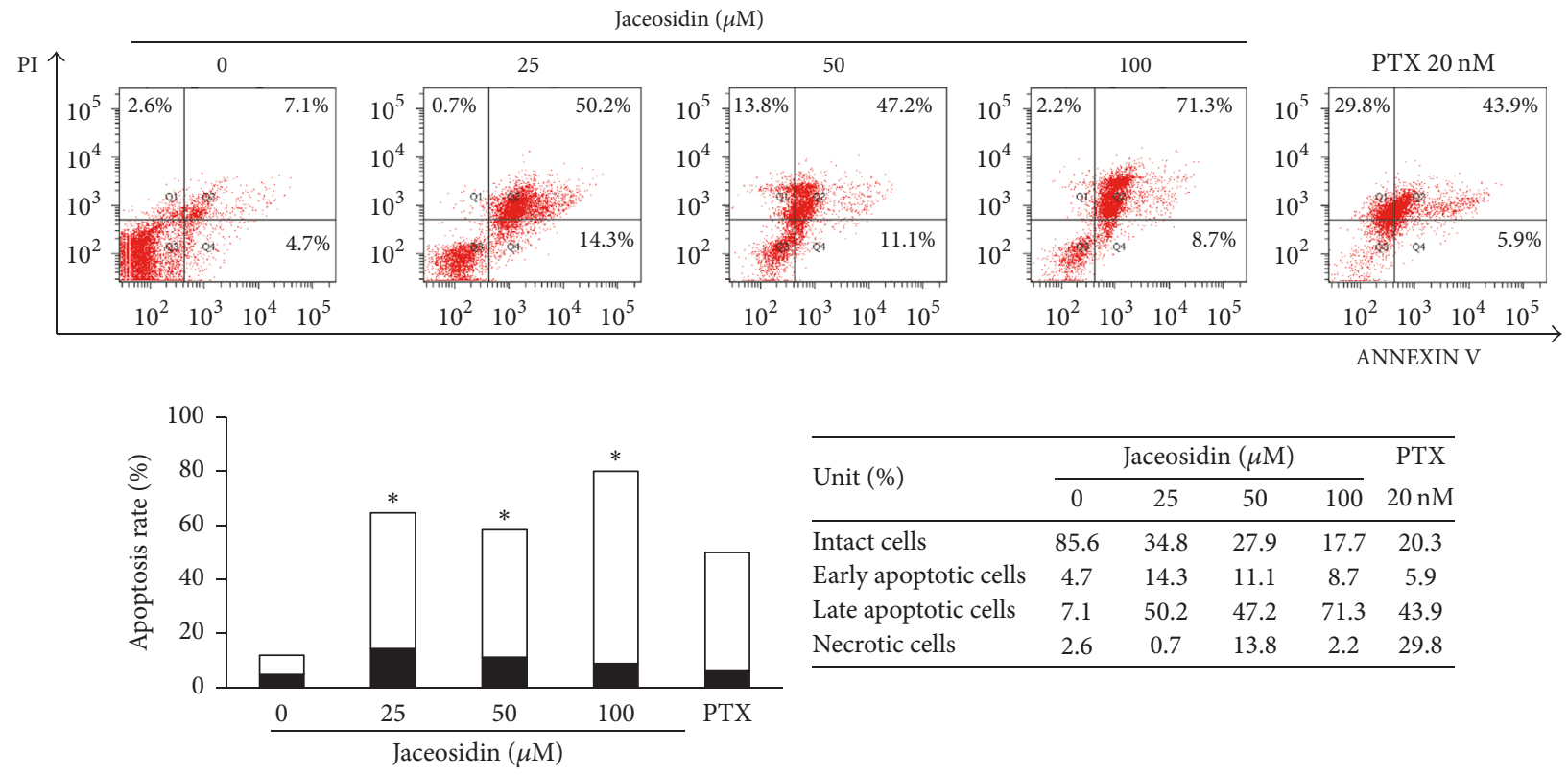

early apoptosis

late apoptosis

(a) HSC-3
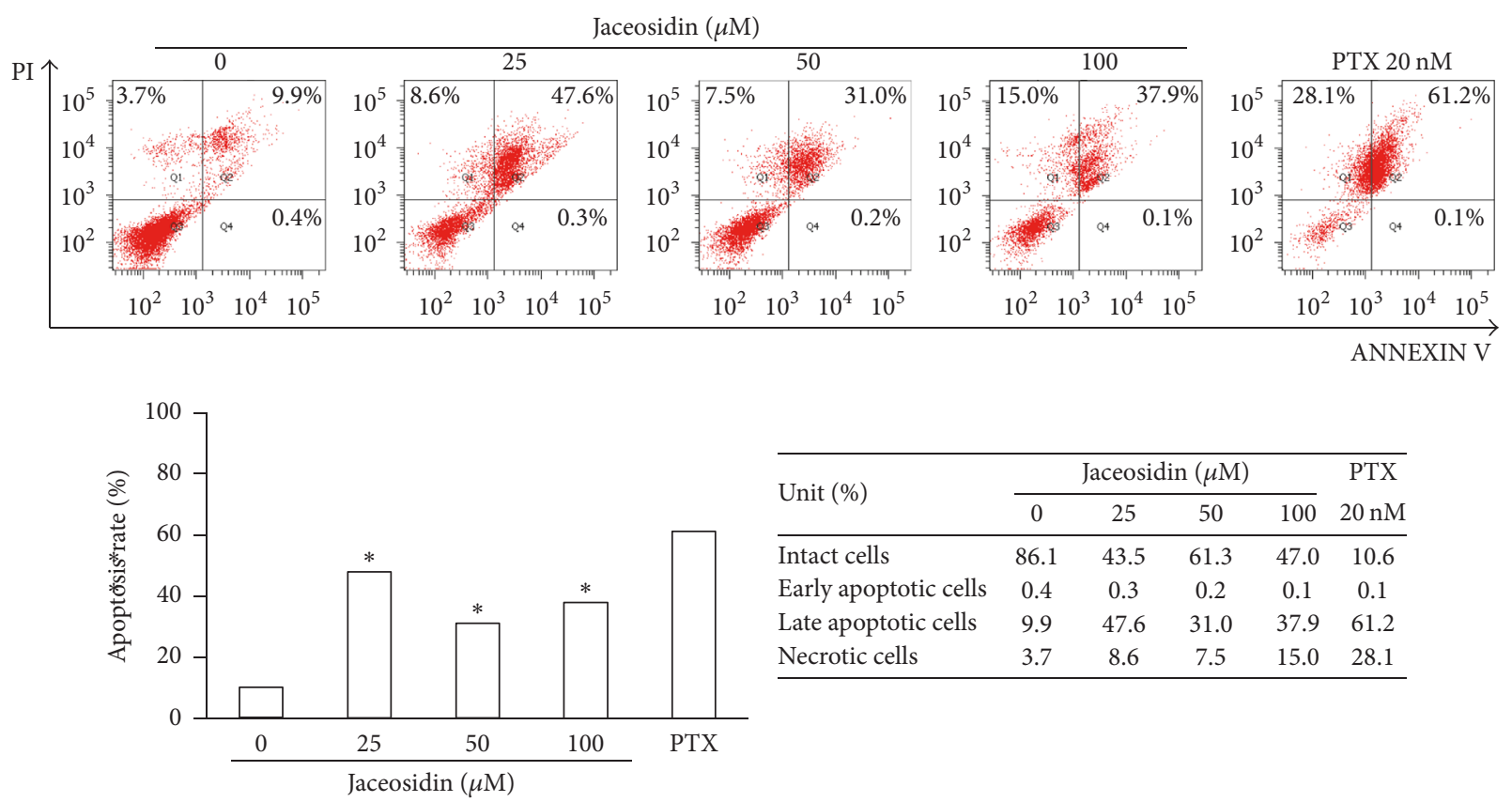

early apoptosis

$\square$ late apoptosis

(b) Ca9.22

FIGURE 2: Jaceosidin induces early and late apoptosis in OSCC cells. Thus, to evaluate jaceosidin-mediated apoptosis, jaceosidin-treated OSCC cells (for $48 \mathrm{~h}$ ) were labelled with PI and Annexin V and apoptotic staining profiles were analysed using flow cytometry $\left({ }^{*} p<0.05\right)$. Paclitaxel (PTX) was used as a positive control; (a) HSC-3 cells; (b) Ca9.22 cells. 


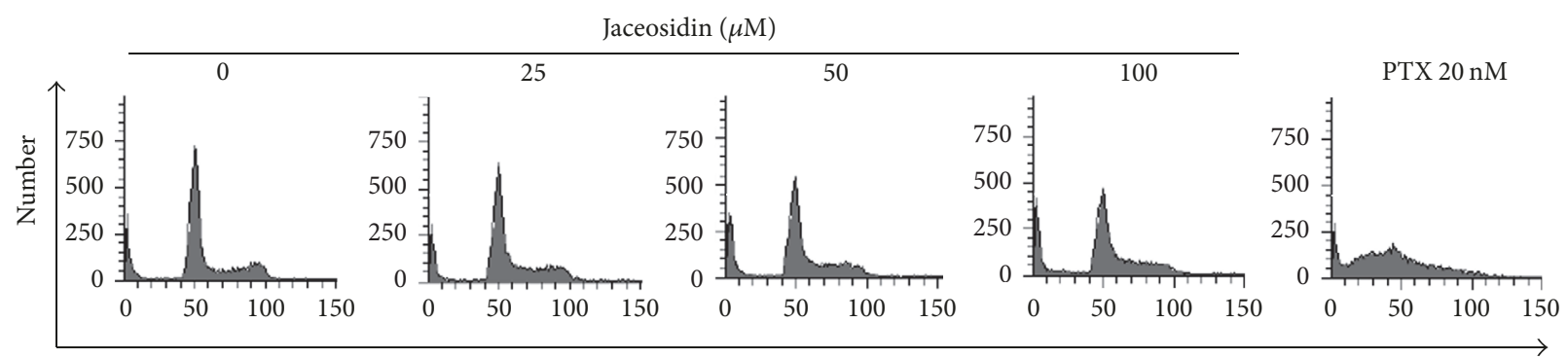

DNA content

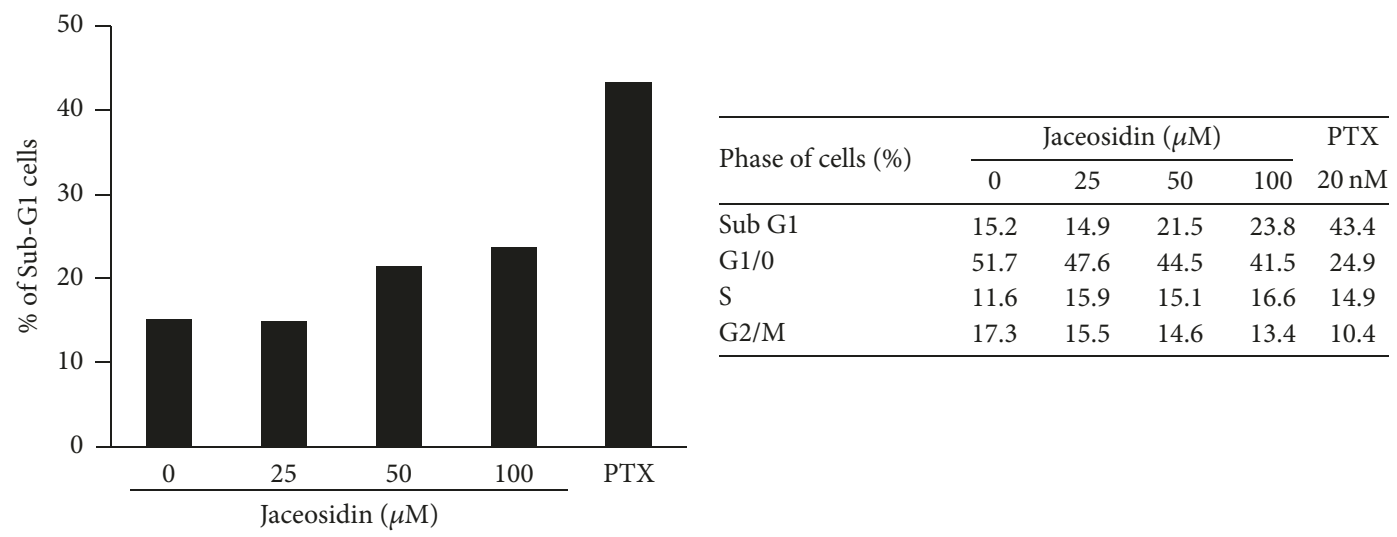

(a) HSC-3
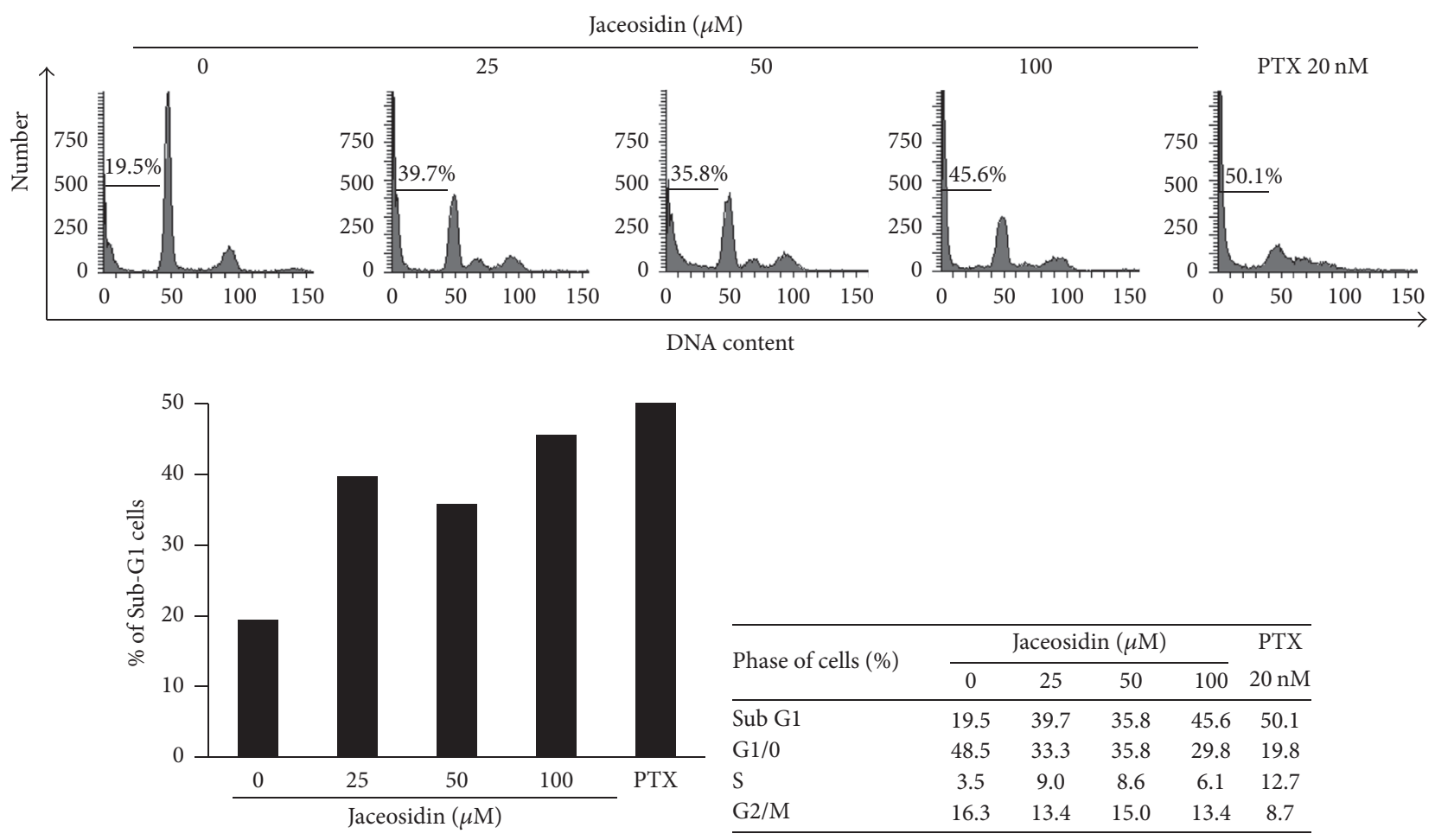

(b) Ca9.22

FIGURE 3: Jaceosidin-treated OSCC cells showed cell cycle arrest at the sub-G1 phase; OSCC cells were cultured in the presence of the indicated jaceosidin concentrations for $48 \mathrm{~h}$ and were stained with propidium iodide (PI). Cell cycle distributions were then evaluated using flow cytometry with paclitaxel (PTX) treatments as a positive control for cell cycle arrest; (a) HSC-3 cells; (b) Ca9.22 cells. 

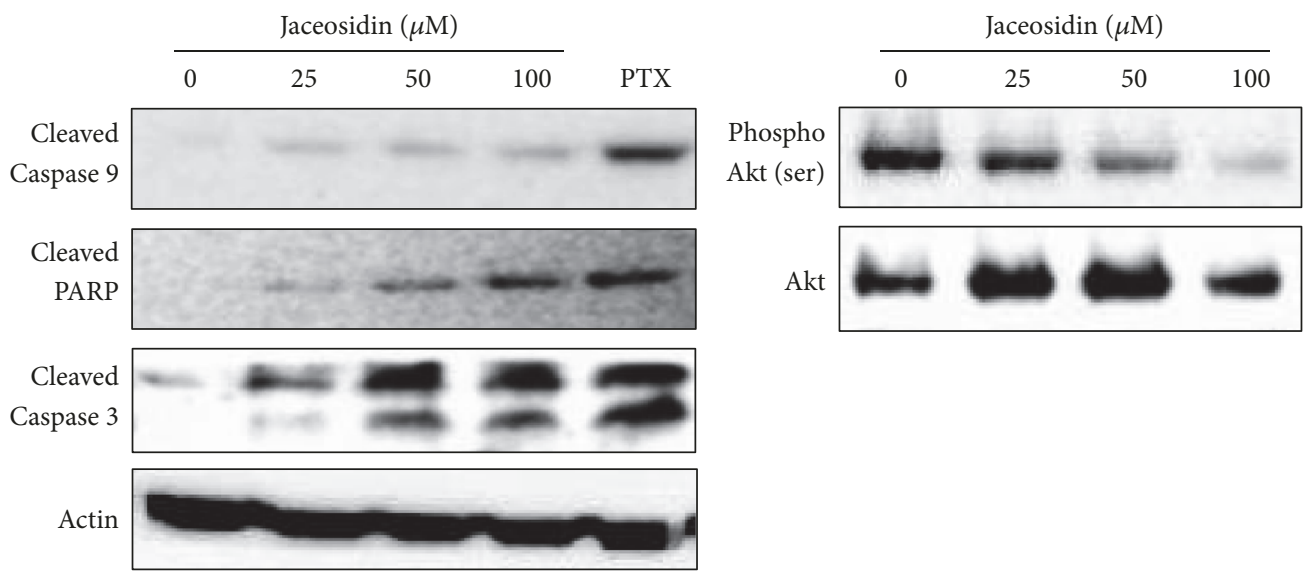

(a) HSC-3
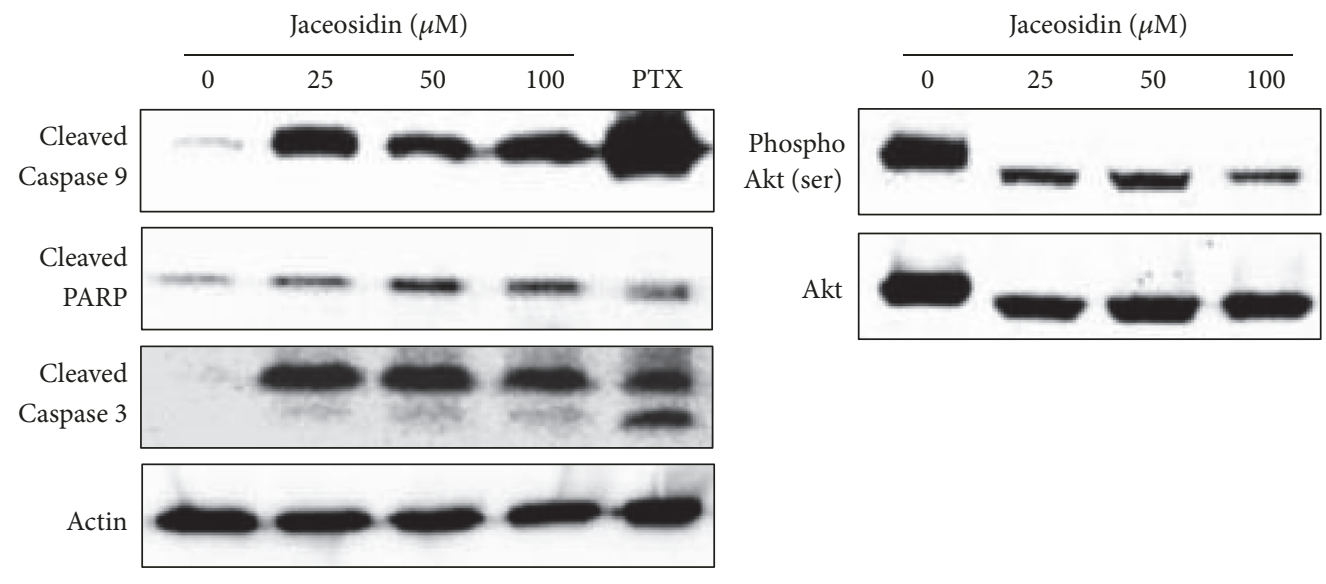

(b) Ca9.22

FIGURE 4: Jaceosidin triggers cleavage of caspase-9, PARP, and caspase-3 and decreases Akt phosphorylation; OSCC cells were treated with the indicated concentrations of jaceosidin for $48 \mathrm{~h}$ and cleaved caspase-9, PARP, and caspase- 3 proteins and phosphorylated Akt were examined using western blotting. Paclitaxel (PTX) was used as a positive control for caspase and PARP cleavage; (a) HSC-3 cells; (b) Ca9.22 cells.

3.3. Jaceosidin Induces Cleavage of Caspase-9, PARP, and Caspase-3 and Inhibits Phosphorylation of Akt. To characterise jaceosidin-mediated apoptotic signalling pathways, we determined the effects on caspase-9, PARP, and caspase-3 cleavage and phosphorylation. In the presence of jaceosidin, caspase-9, PARP, and caspase- 3 cleavage was significantly increased in a dose-dependent manner (Figures 4(a) and 4(b), left). Cleaved caspase-3 is considered a biomarker of apoptosis, and Akt, also known as protein kinase $\mathrm{B}(\mathrm{PKB})$, has well characterised essential roles in tumour cell proliferation and survival [13, 14]. Accordingly, phosphorylation levels of Akt were decreased in jaceosidin-treated OSCC cells (Figure 4(a), right) compared with those in control OSCC cells (Figure 4(b), right), which had sustained high levels of phosphorylated Akt. These data indicate that jaceosidininduced cell death is mediated by caspase pathway activation and blockade of Akt phosphorylation in OSCC cells.

3.4. Jaceosidin Does Not Affect Normal Epithelial Cell Proliferation. To determine the effects of jaceosidin on normal cells, we performed proliferation assays in jaceosidintreated $\mathrm{HaCaT}$ normal epithelial keratinocytes. As shown in
Figure 5(a), jaceosidin did not inhibit $\mathrm{HaCaT}$ cell proliferation at doses of $12.5-100 \mu \mathrm{M}$ and had no effects on Akt phosphorylation (Figure 5(b)), indicating that the cytotoxic effects of jaceosidin are OSCC cancer cell-selective.

3.5. Akt Activation Reversed the Antiproliferative Effects of Jaceosidin in OSCC Cells. To determine the contributions of Akt pathway inactivation to the antiproliferative effects of jaceosidin, we cultured OSCC cells in the presence of $100 \mu \mathrm{M}$ jaceosidin and determined whether the Akt activator SC69 can restore proliferation. As shown in Figure 1, jaceosidin significantly inhibited OSCC cell proliferation at $100 \mu \mathrm{M}$. However, OSCC cell proliferation was dose-dependently restored by SC69 treatments (Figure 6), suggesting that inhibition of the Akt pathway is central to the antiproliferative effects of jaceosidin.

\section{Discussion}

Various natural products have long been considered as sources of anticancer agents, and phytochemicals and other 


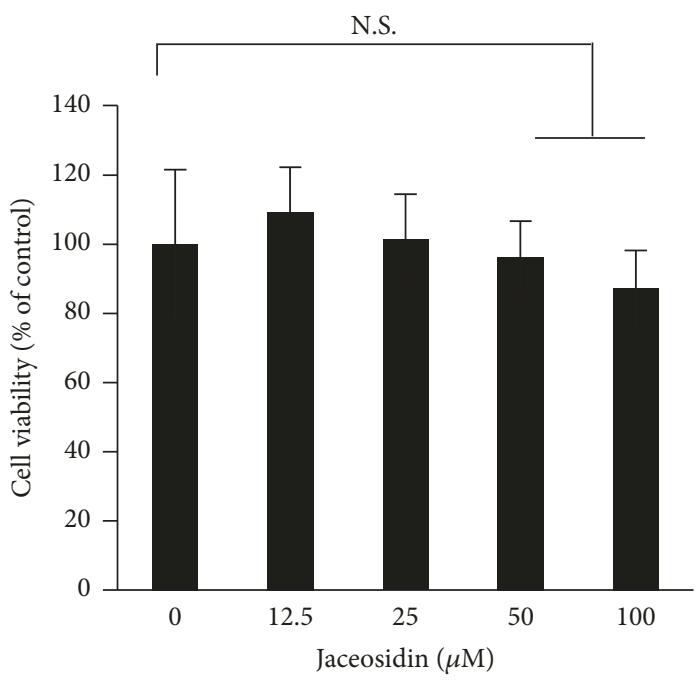

(a)

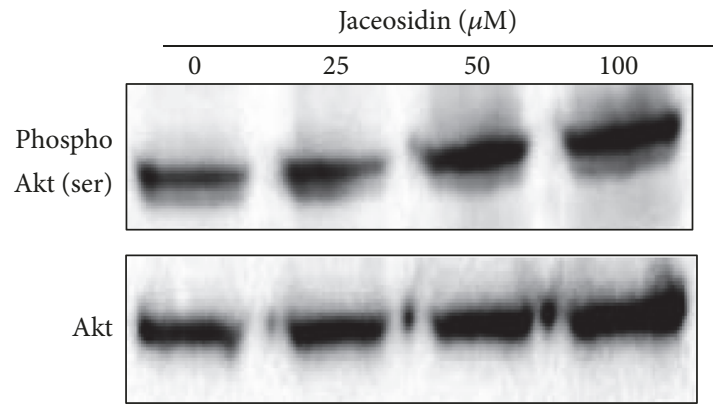

(b)

FIGURE 5: Cytotoxic effects of jaceosidin are absent in normal epithelial keratinocyte cells; (a) HaCaT cells were cultured in the presence of the indicated doses of jaceosidin for $24 \mathrm{~h}$ and cell viability was determined using MTT assays (NS: not significant). (b) HaCaT cells were cultured as in Figure 4 and phosphor-Akt levels were determined using western blotting.

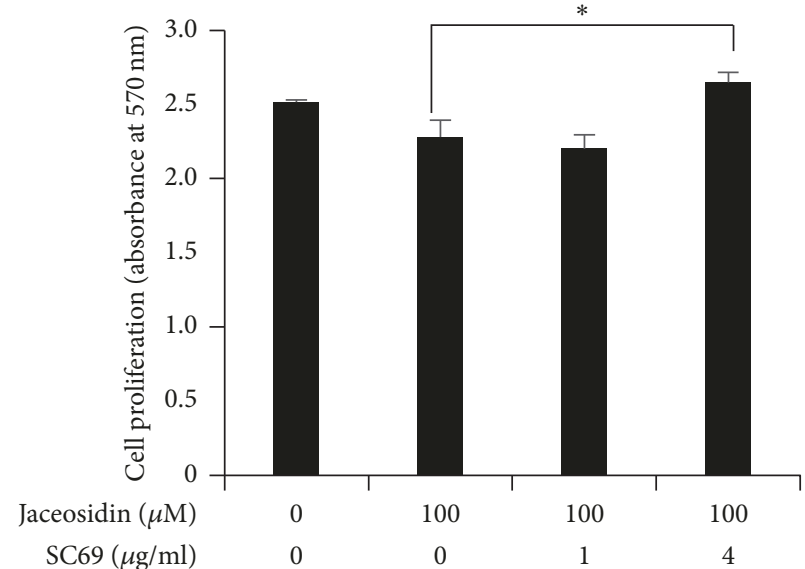

(a) HSC-3

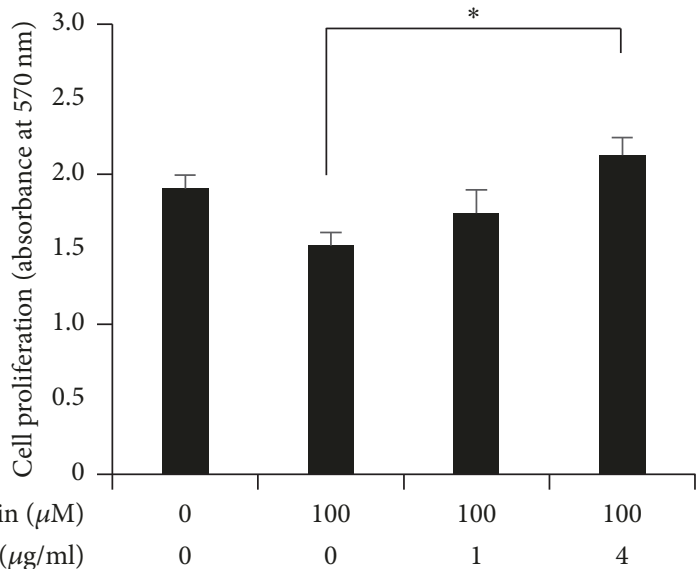

(b) Ca9.22

FIGURE 6: Activation of the Akt pathway reverses the antiproliferative effects of jaceosidin in OSCC cells; OSCC cells were treated with $100 \mu \mathrm{M}$ jaceosidin with the indicated concentrations of the Akt activator SC69 for $24 \mathrm{~h}$ and cell proliferation was determined using MTT assays; ${ }^{*} p<0.05$; (a) HSC-3 cells; (b) Ca9.22 cells.

compounds from plants with food or traditional medicine uses have emerged as promising anticancer treatments and adjuvants. These components can be used to complement or moderate the side effects of anticancer drugs [15]. Moreover, the widely used anticancer agents paclitaxel, curcumin, and evodiamine were originally extracts from herbs and plants [15].

The present data demonstrate that jaceosidin treatments inhibit the proliferation of OSCC cells and cause morphological changes, with no effects on the proliferation of $\mathrm{HaCaT}$ cells at up to $100 \mu \mathrm{M}$, suggesting tumour-specific cytotoxicity. Moreover, the ensuing mechanisms were indicated by increased accumulation of cells in the sub-G1 cell cycle stage, increased presence of early and late apoptotic cells, and cleavage of caspase-9, caspase-3, and PARP proteins. Taken together, these observations show that jaceosidin induces apoptosis of OSCC cells by activating intrinsic apoptotic pathways and also inactivates the Akt pathway.

Previous studies show that jaceosidin affects the proliferation of ovarian, bladder, and endometrial cancer cells [16-18], and most of these reports suggest that anticancer mechanisms of Artemisia extracts involve the induction of apoptosis. Moreover, in agreement with the present observations, Khan et al. showed that jaceosidin-induced apoptosis is accompanied by cell cycle arrest at the G2/M phase in U87 glioblastoma cells [19]. These data suggest that jaceosidin induces 
apoptosis by disrupting the cell cycle. Jaceosidin also reportedly induces apoptosis by altering mitochondrial membrane potential, and Lv et al. showed that jaceosidin induces apoptosis in human ovary cancer cells through mitochondrial pathways [18]. Only one previous report indicates extrinsic and intrinsic pathway activation during jaceosidin-induced apoptosis, suggesting that intrinsic pathways of apoptosis and cell cycle arrest are the primary anticancer mechanisms of jaceosidin.

Importantly, our results demonstrate that jaceosidin-induced cell death is confined to HSC- 3 and Ca9.22 cancer cells, with no growth inhibition of $\mathrm{HaCaT}$ normal keratinocytes at concentrations that were antiproliferative in OSCC cells. In particular, no cytotoxic effects were observed in $\mathrm{HaCaT}$ cells at $\mathrm{IC}_{50}$ of jaceosidin in OSCC cells. The present data are also the first to show that jaceosidin-induced OSCC cell death is apoptotic, and no apoptotic indications were observed in $\mathrm{HaCaT}$ cells in the presence of the same concentrations of jaceosidin.

Apoptosis is regulated by complex caspase cascades, which are extrinsic and intrinsic. Caspase-3 plays key roles in the execution of apoptotic pathways, and as the end point of both intrinsic and extrinsic pathways, cleaved caspase-3 activates PARP and induces apoptosis [20, 21]. Our experiments show that jaceosidin treatment activates caspase- 3 and cleavage of caspase-9, which is activated in the intrinsic apoptosis pathway. Jaceosidin also provoked the cleavage of caspases in HSC3 and Ca9.22 cells, suggesting that jaceosidininduced apoptosis is caspase-dependent.

Although jaceosidin induces apoptosis via the intrinsic pathway, the precise mechanisms remain unclear. The Akt pathway has been associated with cell survival and proliferation in multiple studies, and Akt activation has been correlated with malignant proliferation and evasion of apoptosis in various cancers [22]. Accordingly, the Akt pathway is central to tumour development, and as a key player in apoptotic cascades, it offers a promising therapeutic target for the treatment of cancer $[13,23]$. Jaceosidin treatments effectively inhibited Akt phosphorylation in the present OSCC cell lines, and ectopic activation of Akt diminished the proapoptotic effects of jaceosidin (Figure 6). Collectively, these results indicate that jaceosidin-induced apoptosis in OSCC cells is in part mediated by inactivation of the Akt pathway.

\section{Conclusion}

The present observations demonstrate that jaceosidin selectively inhibits OSCC cell proliferation by inducing caspasedependent apoptosis and inhibiting the Akt pathway. We also clarified the modes of action of jaceosidin in OSCC cells and suggested that further consideration of jaceosidin as a promising new chemotherapeutic agent for the treatment of OSCC is warranted.

\section{Conflicts of Interest}

The authors declare no conflicts of interest regarding the publication of this paper.

\section{Authors' Contributions}

Hye-Yeon Han and Hyung Joon Kim contributed equally to this work.

\section{Acknowledgments}

This work was supported by the National Research Foundation of Korea (NRF) grant funded by the Korean government (MSIP) (NRF-2015R1D1A1A01060617 and NRF2016R1C1B2012891).

\section{References}

[1] K.-W. Jung, Y.-J. Won, H.-J. Kong, C.-M. Oh, D. H. Lee, and J. S. Lee, "Cancer statistics in Korea: incidence, mortality, survival and prevalence in 2011," Cancer Research and Treatment, vol. 46, no. 2, pp. 109-123, 2014.

[2] W. Jerjes, T. Upile, A. Petrie et al., "Clinicopathological parameters, recurrence, locoregional and distant metastasis in 115 T1-T2 oral squamous cell carcinoma patients," Head \& Neck Oncology, vol. 2, no. 1, article no. 9, 2010.

[3] M. Mollaoğlu and G. Erdoğan, "Effect on symptom control of structured information given to patients receiving chemotherapy," European Journal of Oncology Nursing, vol. 18, no. 1, pp. 78-84, 2014.

[4] R. S. Y. Wong, "Apoptosis in cancer: from pathogenesis to treatment," Journal of Experimental \& Clinical Cancer Research, vol. 30, no. 1, article 87, 2011.

[5] E. Y. Park, K.-W. Lee, H.-W. Lee et al., “The ethanol extract from Artemisia princeps Pampanini induces p53-mediated G1 phase arrest in A172 human neuroblastoma cells," Journal of Medicinal Food, vol. 11, no. 2, pp. 237-245, 2008.

[6] K.-S. Chung, J.-H. Choi, N.-I. Back et al., "Eupafolin, a flavonoid isolated from Artemisia princeps, induced apoptosis in human cervical adenocarcinoma HeLa cells," Molecular Nutrition \& Food Research, vol. 54, no. 9, pp. 1318-1328, 2010.

[7] M. J. Kim, J. M. Han, Y. Y. Jin et al., "In vitro antioxidant and anti-inflammatory activities of jaceosidin from Artemisia princeps Pampanini cv. Sajabal," Archives of Pharmacal Research, vol. 31, no. 4, pp. 429-437, 2008.

[8] D. H. Na, "Metabolism study of botanical drugs," Archives of Pharmacal Research, vol. 33, no. 12, pp. 1877-1879, 1877.

[9] W. Y. Song, H. Y. Ji, N.-I. Baek, T.-S. Jeong, and H. S. Lee, "In Vitro metabolism of Jaceosidin and characterization of cytochrome P450 and UDP-glucuronosyltransferase enzymes in human liver microsomes," Archives of Pharmacal Research, vol. 33, no. 12, pp. 1985-1996, 2010.

[10] T. H. Lee, H. Jung, K. H. Park, M. H. Bang, N.-I. Baek, and J. Kim, "Jaceosidin, a natural flavone, promotes angiogenesis via activation of VEGFR2/FAK/PI3K/AKT/NF-iB signaling pathways in endothelial cells," Experimental Biology and Medicine, vol. 239, no. 10, pp. 1325-1334, 2014.

[11] H.-G. Lee, K.-A. Yu, W.-K. Oh et al., "Inhibitory effect of jaceosidin isolated from Artemisiaargyi on the function of E6 and E7 oncoproteins of HPV 16," Journal of Ethnopharmacology, vol. 98, no. 3, pp. 339-343, 2005.

[12] K.-S. Chung, H.-E. Choi, J.-S. Shin et al., "Chemopreventive effects of standardized ethanol extract from the aerial parts of Artemisia princeps Pampanini cv. Sajabal via NF- $\kappa$ B inactivation 
on colitis-associated colon tumorigenesis in mice," Food and Chemical Toxicology, vol. 75, pp. 14-23, 2015.

[13] G. M. Nitulescu, D. Margina, P. Juzenas et al., "Akt inhibitors in cancer treatment: The long journey from drug discovery to clinical use (Review)," International Journal of Oncology, vol. 48, no. 3, pp. 869-885, 2016.

[14] F. Liang and S. Xie, "Puerarin prevents tumor necrosis factor- $\alpha$ induced apoptosis of PC12 cells via activation of the Pi3K/Akt signaling pathway," Experimental and Therapeutic Medicine, vol. 14, no. 1, pp. 813-818, 2017.

[15] L. Shu, K. Cheung, T. O. Khor, C. Chen, and A. Kong, "Phytochemicals: cancer chemoprevention and suppression of tumor onset and metastasis," Cancer and Metastasis Reviews, vol. 29, no. 3, pp. 483-502, 2010.

[16] A. J. Min, W. L. Ki, D.-Y. Yoon, and J. L. Hyong, "Jaceosidin, a pharmacologically active flavone derived from Artemisia argyi, inhibits phorbol-ester-induced upregulation of COX-2 and MMP-9 by blocking phosphorylation of ERK-1 and -2 in cultured human mammary epithelial cells," Annals of the New York Academy of Sciences, vol. 1095, pp. 458-466, 2007.

[17] J.-G. Lee, J.-H. Kim, J.-H. Ahn, K.-T. Lee, N.-I. Baek, and J.H. Choi, "Jaceosidin, Isolated from dietary mugwort (Artemisia princeps), Induces $\mathrm{G} 2 / \mathrm{M}$ cell cycle arrest by inactivating cdc25C-cdc2 via ATM-Chk1/2 activation," Food and Chemical Toxicology, vol. 55, pp. 214-221, 2013.

[18] W. Lv, X. Sheng, T. Chen, Q. Xu, and X. Xie, "Jaceosidin induces apoptosis in human ovary cancer cells through mitochondrial pathway," Journal of Biomedicine and Biotechnology, vol. 2008, Article ID 394802, 2008.

[19] M. Khan, B. Yu, A. Rasul et al., "Jaceosidin induces apoptosis in U87 glioblastoma cells through G2/M phase arrest," EvidenceBased Complementary and Alternative Medicine, vol. 2012, Article ID 703034, 12 pages, 2012.

[20] Y. Kiraz, A. Adan, M. Kartal Yandim, and Y. Baran, "Major apoptotic mechanisms and genes involved in apoptosis," Tumor Biology, vol. 37, no. 7, pp. 8471-8486, 2016.

[21] A. Rasul, B. Yu, M. Khan et al., "Magnolol, a natural compound, induces apoptosis of SGC-7901 human gastric adenocarcinoma cells via the mitochondrial and PI3K/Akt signaling pathways," International Journal of Oncology, vol. 40, no. 4, pp. 1153-1161, 2012.

[22] A. Rasul, M. Khan, B. Yu et al., "Isoalantolactone, a sesquiterpene lactone, induces apoptosis in SGC-7901 cells via mitochondrial and phosphatidylinositol 3-kinase/Akt signaling pathways," Archives of Pharmacal Research, vol. 36, no. 10, pp. 1262-1269, 2013.

[23] A. Rasul, C. Ding, X. Li et al., "Dracorhodin perchlorate inhibits PI3K/Akt and NF- $\kappa$ B activation, up-regulates the expression of p53, and enhances apoptosis," Apoptosis, vol. 17, no. 10, pp. 11041119, 2012. 


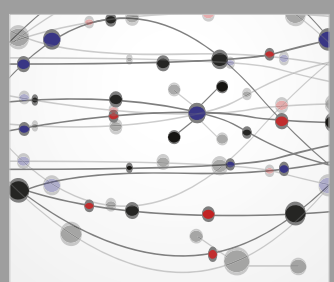

The Scientific World Journal
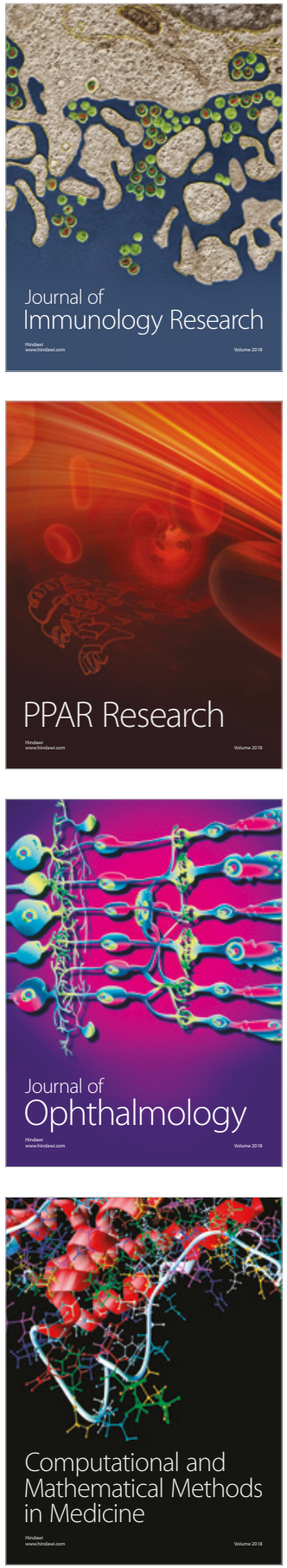

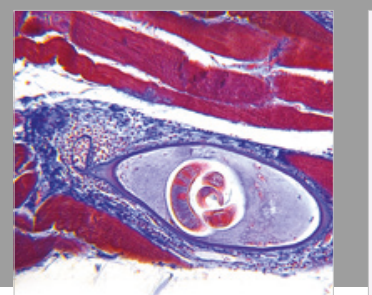

Gastroenterology Research and Practice

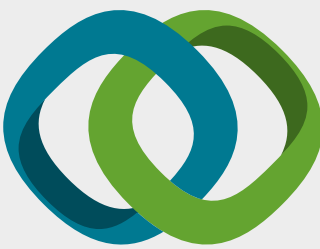

\section{Hindawi}

Submit your manuscripts at

www.hindawi.com
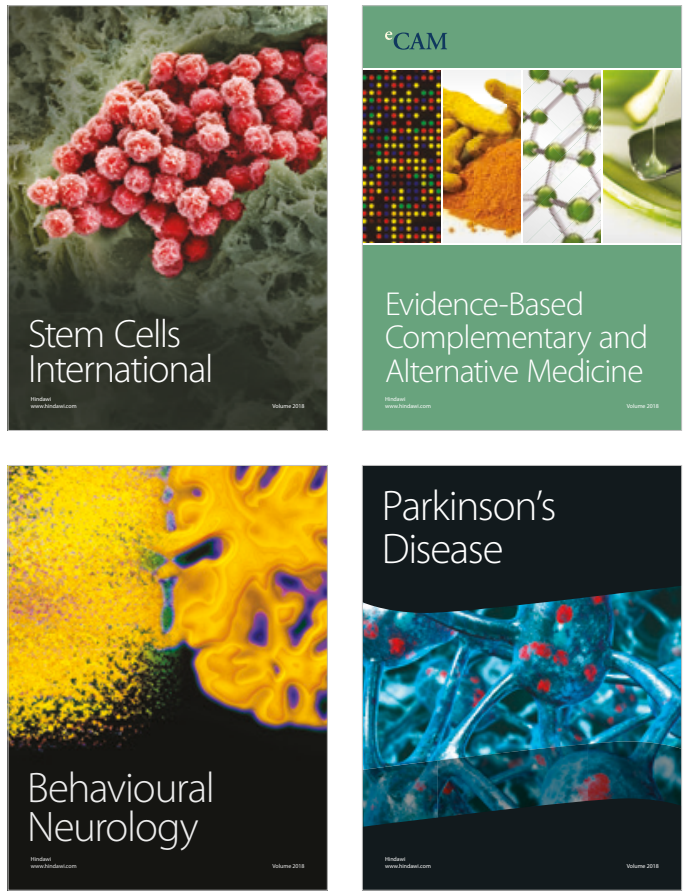

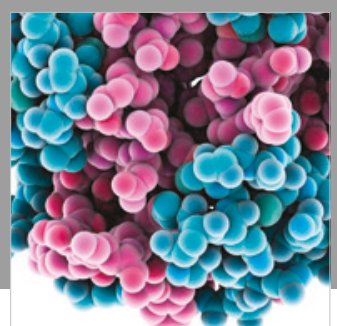

ournal of

Diabetes Research

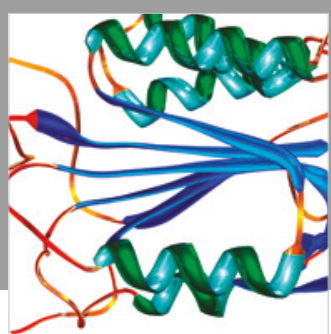

Disease Markers
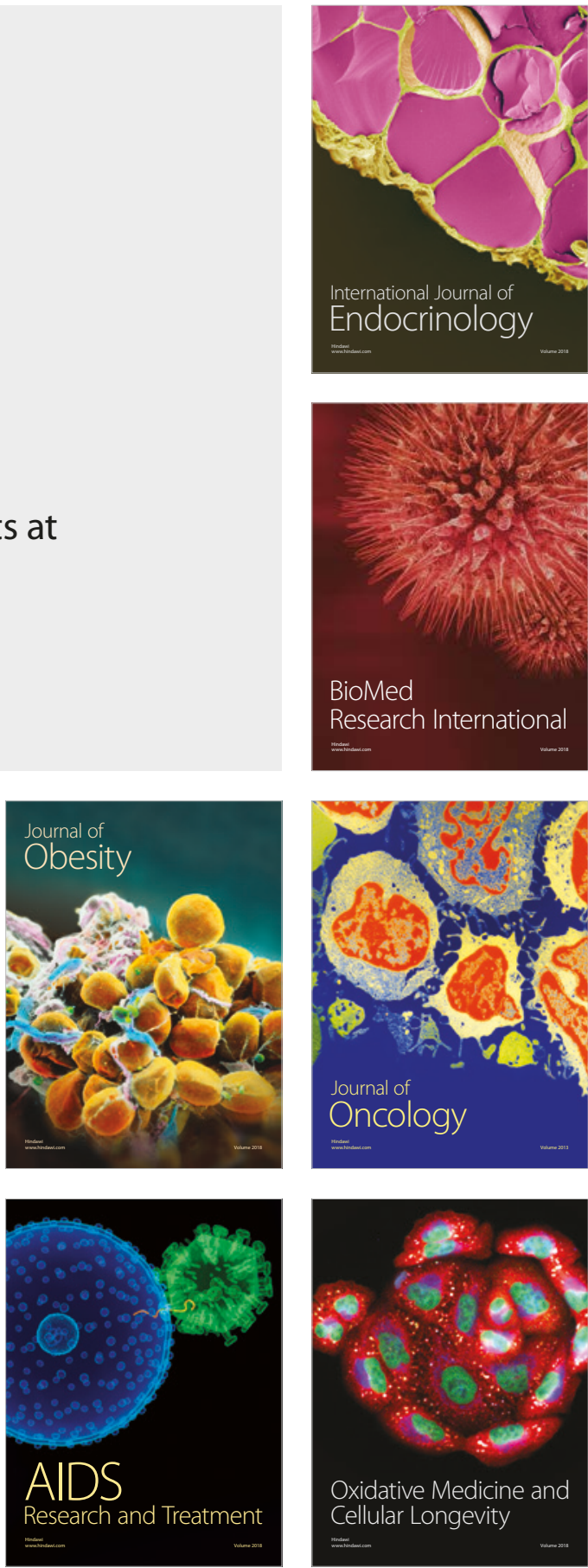